\title{
Production of excited atomic hydrogen and deuterium from $\mathrm{H}_{2}$ and $\mathrm{D}_{2}$ photodissociation
}

\author{
J D Bozek ${ }^{1}$, J E Furst ${ }^{2}$, T J Gay ${ }^{3,7}$, H Gould ${ }^{1}$, A L D Kilcoyne ${ }^{1}$, \\ J R Machacek ${ }^{3}$, F Martín ${ }^{4}$, K W McLaughlin ${ }^{5}$ and J L Sanz-Vicario ${ }^{4,6}$ \\ ${ }^{1}$ Lawrence Berkeley National Laboratory, Berkeley, CA 94720, USA \\ 2 University of Newcastle-Ourimbah, Ourimbah, NSW 2258, Australia \\ ${ }^{3}$ Behlen Laboratory of Physics, University of Nebraska, Lincoln, NE 68588-0111, USA \\ ${ }^{4}$ Departamento de Química, Universidad Autónoma de Madrid, 28049 Madrid, Spain \\ ${ }^{5}$ Department of Physics and Engineering, Loras College, Dubuque, IA 52001, USA \\ ${ }^{6}$ Instituto de Física, Universidad de Antioquia, Medellín, Colombia
}

Received 21 June 2006, in final form 17 September 2006

Published 10 November 2006

Online at stacks.iop.org/JPhysB/39/4871

\begin{abstract}
We have measured the production of both $\mathrm{Ly} \alpha$ and $\mathrm{H} \alpha$ fluorescence from atomic $\mathrm{H}$ and $\mathrm{D}$ for the photodissociation of $\mathrm{H}_{2}$ and $\mathrm{D}_{2}$ by linearly polarized photons with energies between 24 and $60 \mathrm{eV}$. In this energy range, excited photofragments result primarily from the production of doubly excited molecular species which promptly autoionize or dissociate into two neutrals. Our data are compared with ab initio calculations of the dissociation process, in which both doubly excited state production and prompt ionization (nonresonant) channels are considered. Agreement between our experimental data and that of earlier work, and with our theoretical calculations, is qualitative at best.
\end{abstract}

(Some figures in this article are in colour only in the electronic version)

\section{Introduction}

Photodissociation of $\mathrm{H}_{2}$ is the simplest chemical reaction, yet our theoretical understanding of it is incomplete [1-7]. The photodissociation process involves the correlated motion of strongly interacting particles, often having large potential and/or kinetic energy. As such, it serves as a prototype of a many-body system far from equilibrium. Photodissociation of $\mathrm{H}_{2}$ by VUV photons is important in a host of important applied problems as well, including fusion plasmas [8], interstellar and intergalactic media [9] and the dynamics of extrasolar planetary atmospheres [10].

Molecular hydrogen can be dissociated by photon impact in four distinct ways:

$$
\gamma+\mathrm{H}_{2} \rightarrow \mathrm{H}_{2}^{*} \rightarrow \mathrm{H}(1)+\mathrm{H}(n),
$$

7 Author to whom correspondence should be addressed. 


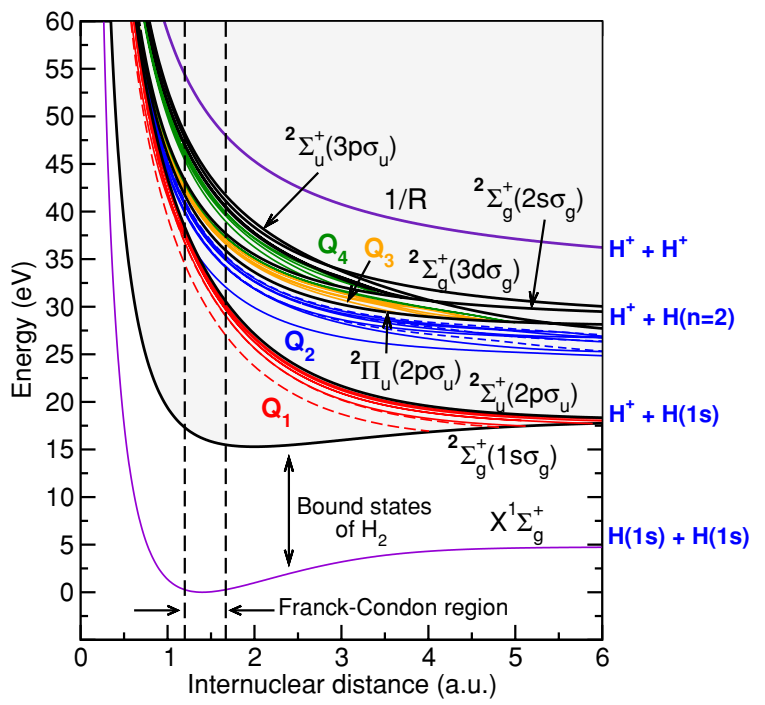

Figure 1. Potential energy curves of $\mathrm{H}_{2}$ and $\mathrm{H}_{2}^{+}$showing the doubly excited $\mathrm{Q}$ states (see [5] and references therein). Thin dashed curves: $\mathrm{Q}$ states of ${ }^{1} \Sigma_{\mathrm{u}}^{+}$symmetry; thin continuous curves: Q states of ${ }^{1} \Pi_{\mathrm{u}}$ symmetry. Thick curves: $\mathrm{H}_{2}^{+}$states. A large number of singly excited bound states of $\mathrm{H}_{2}$ exist in the region bounded by the $\mathrm{H}_{2}$ and $\mathrm{H}_{2}^{+}$ground states, as indicated.

$$
\begin{aligned}
\rightarrow \mathrm{H}_{2}^{+*}+ & \mathrm{e}^{-} \rightarrow \mathrm{H}(n)+\mathrm{H}^{+}+\mathrm{e}^{-}, \\
\rightarrow \mathrm{H}_{2}^{* *} & \rightarrow \mathrm{H}_{2}^{+*}+\mathrm{e}^{-} \rightarrow \mathrm{H}(n)+\mathrm{H}^{+}+\mathrm{e}^{-}, \\
& \rightarrow \mathrm{H}^{-}+\mathrm{H}^{+}, \\
& \rightarrow \mathrm{H}(n)+\mathrm{H}^{+}+\mathrm{e}^{-}, \\
& \rightarrow \mathrm{H}(n)+\mathrm{H}\left(n^{\prime}\right),
\end{aligned}
$$

and

$$
\rightarrow 2 \mathrm{H}^{+}+2 \mathrm{e}^{-} .
$$

Processes (1), (2), (3) and (4) correspond, respectively, to the production of a singly excited molecule which subsequently dissociates, direct non-resonant molecular ionization followed by prompt dissociation, production of doubly excited 'Q' states embedded in the continuum which can either autoionize and dissociate or promptly dissociate into a variety of possible photofragments and, finally, prompt double ionization. Understanding processes (1)-(4) is aided by reference to figure 1 . Since photon absorption is practically an instantaneous process, the molecular target can be thought of as making a 'vertical (Franck-Condon) transition', whose height corresponds to the incident photon energy. However, the subsequent evolution of the system through processes (1)-(4) implies that the electronic and nuclear motions cannot be considered separately, and that the assumption of adiabatic nuclear motion during dissociation is no longer appropriate.

While the photodissociation of $\mathrm{H}_{2}$ is fundamental, it is not simple, as is apparent from the cappellini-like potential energy diagram of figure 1. In this paper, we concentrate on processes involving two active electrons that result in at least one neutral photofragment, i.e. (2) and (3) above. These channels cannot be considered in an independent electron 
model and as such are particularly sensitive to correlated electron motion. (The direct double ionization channel has recently been investigated using a combination of state-ofthe-art experimental and calculational tools [7, 11].) When the incident photon energy $E$ exceeds $\sim 26 \mathrm{eV}$, the lowest lying doubly excited states, corresponding to a $2 \mathrm{p} \sigma_{\mathrm{u}} \mathrm{H}_{2}^{+}$state core dressed with an excited electron, become accessible. Since the corresponding potential energy curves are repulsive, autoionization of these states (which can be slow) competes with dissociation into neutrals, which means that the autoionization lifetime is also sensitive to the nuclear motion, and interferences between ionization and dissociation may occur. The Q states that do not autoionize (i.e. Q states with an autoionization lifetime larger than the dissociation time) dissociate diabatically. In the region above $26 \mathrm{eV}$, population of higher lying doubly excited and $\mathrm{H}_{2}^{+}$states and, finally, double ionization, are the only mechanisms for photofragment production. Above $\sim 54 \mathrm{eV}$, only single or double direct ionization can produce photofragments, because resonant production of the $\mathrm{Q}$ states can occur only below the $(1 / R)$ Coulombic $\mathrm{H}^{+}+\mathrm{H}^{+}+2 \mathrm{e}^{-}$asymptotic limit. Photofragments produced either through intermediate $\mathrm{H}_{2}^{+}$or $\mathrm{H}_{2}^{* *}$ states are 'fast', with kinetic energies $>2.5 \mathrm{eV}$, coming as they do from potential energy curves that are strongly repulsive in the Franck-Condon region.

Complete unraveling of the breakup paths in reactions (2) and (3) dictates quantumstate-specific identification of the photofragments. This can be accomplished effectively by observing fluorescence from the decay of the excited $\mathrm{H}$. The simplest and most elementary measurements of this type are 'excitation functions' for the photofragments, i.e., the measurement of fluorescent intensity versus $E$. Given the ubiquity and broad importance of $\mathrm{H}_{2}$, the paucity of such data is surprising. The only excitation function studies for channels (2) and (3) of which we are aware have been carried out by Glass-Maujean and co-workers [12-16], Arai et al [17] and Odagiri et al [18]. Experiments to date have investigated Ly $\alpha$, $\mathrm{H} \alpha, \mathrm{H} \beta$ and $\mathrm{H} \gamma$ emissions, with $E$ ranging between $\sim 26$ and $60 \mathrm{eV}$. (Below, we will use the abbreviations 'Ly $\alpha$ ' for Lyman- $\alpha, n=2 \rightarrow n=1$ emission at $122 \mathrm{~nm}$, ' $\mathrm{H} \alpha$ ' for Balmer- $\alpha$, $n=3 \rightarrow n=2$ emission at $656 \mathrm{~nm}$, and ' $\mathrm{H} \beta$ ' for Balmer- $\beta, n=4 \rightarrow n=2$ emission at $486 \mathrm{~nm}$.)

Agreement between these data and the only existing theoretical calculations has been poor. As mentioned above, photodissociation from doubly excited states competes with ionization and autoionization. However, previous attempts [3, 4, 6, 13-16] to evaluate the photodissociation cross section $\sigma_{\mathrm{d}}$ have made use of the simple empirical formula $\sigma_{\mathrm{d}}(E)=\chi_{\mathrm{d}} \sigma_{\mathrm{a}}(E)$, where $\sigma_{\mathrm{a}}$ is the absorption cross section evaluated in the Franck-Condon approximation and $\chi_{\mathrm{d}}$ is the dissociation yield or survival probability. The latter quantity has been either estimated [13] or calculated semiclassically [3, 4] assuming that it is independent of the photon energy. This procedure neglects interferences between autoionization and direct ionization, as well as between different doubly excited states.

A proper theoretical treatment that includes the competition between all possible ionization and dissociation channels has recently become available [19]. It involves solving the time-dependent Schrödinger equation in a basis of molecular Feshbach states especially designed to describe resonant and non-resonant processes as well as interferences between them. B-spline basis functions are used to represent both the electronic and the nuclear wavefunctions. The method takes into account all electronic and vibrational degrees of freedom and, therefore, allows one to describe the interplay between electronic and nuclear motions. It has been successfully used to describe dissociative photoionization and photodissociation of $\mathrm{H}_{2}$ up to $\sim 36 \mathrm{eV}$ [19].

In this paper, we report new measurements of $\mathrm{Ly} \alpha$ and $\mathrm{H} \alpha$ fluorescence excitation functions from 24 to $60 \mathrm{eV}$ for $\mathrm{H}_{2}$ and $\mathrm{D}_{2}$, in combination with the results of the new 
theory, which has been extended to account for $\mathrm{D}_{2}$ and a substantially wider photon energy range.

\section{Experimental details}

Our measurements were performed on the high-resolution atomic, molecular and optical physics undulator beam line (10.0.1.2) of the Advanced Light Source (ALS) at the Lawrence Berkeley National Laboratory. A grazing-incidence spherical-grating monochromator equipped with a 380 lines $\mathrm{mm}^{-1}$ grating was used to select values of $E$ between 26 and $60 \mathrm{eV}$ with a constant resolution of $30 \mathrm{meV}$. The light from the monochromator had a linear polarization in the horizontal plane $>99 \%$. This light was collimated to a beam spot size of $\sim 0.5 \mathrm{~mm}$, at which point it intersected an effusive target of $\mathrm{H}_{2}$ or $\mathrm{D}_{2}$ gas. Downstream from the interaction region, its intensity was monitored by a calibrated photodiode (IRD AXUV100). The collision chamber and fluorescence detection apparatus have been described previously [20], but both were modified from the original set-up as described below.

The photon-gas collision region was observed by optical detection systems at polar angles of $35.3^{\circ}, 144.7^{\circ}$ and $90^{\circ}$ relative to the incident photon propagation axis, the first two being in the plane defined by the electric field of the incident photons and their propagation axis, the latter being perpendicular to this plane. The photon detectors at $35.3^{\circ}$ and $90^{\circ}$ were used to detect $\mathrm{H} \alpha$ light and comprised $f / 1.9$ fused-silica collection lenses, a linear polarizer in the case of the $90^{\circ}$ port to eliminate polarization sensitivity of the fluorescence intensity [21], interference filters $(656 \mathrm{~nm} ; 0.9 \mathrm{~nm}$ FWHM) to select the atomic transition to be monitored and lenses to refocus the collimated light onto the GaAs photocathodes of the photomultiplier tubes (Hamamatsu R943-02) used to detect the light. Titanium cones with axes that were coincident with the centre of the interaction region were used to shield the insulating surfaces of the focusing lenses. The front ends of these cones were $36 \mathrm{~mm}$ from the intersection point defined by the axes of the photon beam and the effusive gas jet needle, whose tip was $\sim 2 \mathrm{~mm}$ from this point. Ly $\alpha$ photons were detected by a channel electron multiplier preceded by a $\mathrm{MgF}_{2}$ window which limited this detector's sensitivity to photons with wavelengths between 115 and $200 \mathrm{~nm}$. Being at 'magic angles', neither this detector nor the one at $35.3^{\circ}$ required polarization correction.

In order to determine the possible effects of radiation trapping and quenching of excited $\mathrm{H}$ atoms by $\mathrm{H}_{2}$ [22], we studied the pressure dependence of the shapes of the intensity curves. No dependence was observed between a chamber pressure of $2 \times 10^{-4}$ and $2 \times 10^{-6}$ Torr. From the data of Humphrey [23], and factoring in the gas sensitivity of our Bayard-Alpert ionization gauge, we estimate that the gauge pressure range indicated corresponds to an interaction region pressure from $7 \mathrm{~m}$ Torr to $1.5 \mathrm{mTorr}$. This is consistent with negligible radiation trapping or quenching.

\section{Theoretical calculations}

In the theoretical calculations we discuss here, we solve the time-dependent Schrödinger equation (TDSE) using the exact non-relativistic Hamiltonian of $\mathrm{H}_{2}$ and the radiation-molecule interaction potential in the dipole approximation and velocity gauge, $V(t)=\vec{p} \cdot \vec{A}(t)$. For a photon energy $\hbar \omega$, the vector potential $\vec{A}(t)$, polarized along the vector $\vec{e}_{z}$, is defined in a finite time interval $[-T / 2,+T / 2]$ as $\vec{A}(t)=A_{0} f(t) \cos (\omega t+\varphi)$, with an envelope $f(t)=\cos ^{2}(\pi t / T)$ and a phase $\varphi=0$. Since the experiments are performed with synchrotron radiation, one must define $\vec{A}(t)$ in a long enough time interval. We have used $T=10 \mathrm{fs}$, but 
we have checked that longer intervals lead to practically identical results [19]. Following the usual Feshbach procedure in which the non-resonant and resonant parts of the electronic wavefunction are assigned to two orthogonal complementary subspaces $P$ and $Q$, respectively, we project the TDSE in a basis of molecular vibronic states associated with each subspace (see [19] for details). These states are written in the Born-Oppenheimer approximation as products of electronic and vibrational wavefunctions. The bound electronic wavefunctions are represented in a basis of configurations built from $\mathrm{H}_{2}^{+}$orbitals expanded in a basis of spherical harmonics and a basis of radial B-splines defined in a box of finite size. The continuum states are evaluated by means of the $\mathrm{L}^{2}$ close-coupling method [24] by using the same $\mathrm{H}_{2}^{+}$ orbitals and B-spline functions as for bound states. The 'discretized' continuum states lead to total photoionization cross sections that are practically identical to the experimental ones [25]. Vibrational (bound and dissociative) wavefunctions have also been expanded in a basis of B-splines. The size of the electronic and nuclear boxes has been chosen so that the spacing between discretized levels is much smaller than the photon bandwidth and the autoionization widths of the relevant doubly excited states. These conditions ensure that the electronic and vibrational wave packets do not reach the walls of the electronic and vibrational boxes at $t \leqslant$ $+T / 2$ and that the autoionization decay is properly described [19].

The calculations reported here include the ground state of $\mathrm{H}_{2}\left(\mathrm{D}_{2}\right)$ and all the vibronic states (dissociative and non-dissociative) with energies below $\hbar \omega+6.0 \mathrm{eV}$ associated with the lowest six Q1, seven Q2 and six Q3 doubly excited states of both ${ }^{1} \Sigma_{\mathrm{u}}^{+}$and ${ }^{1} \Pi_{\mathrm{u}}$ symmetries [26-28] and with the $1 \mathrm{~s} \sigma_{\mathrm{g}} \varepsilon l, 2 \mathrm{p} \sigma_{\mathrm{u}} \varepsilon l, 2 \mathrm{p} \pi_{\mathrm{u}} \varepsilon l, 2 \mathrm{~s} \sigma_{\mathrm{g}} \varepsilon l, 3 \mathrm{p} \sigma_{\mathrm{u}} \varepsilon l, 3 \mathrm{~d} \sigma_{\mathrm{g}} \varepsilon l, 3 \mathrm{~d} \pi_{\mathrm{g}} \varepsilon l, 3 \mathrm{~d} \delta_{\mathrm{g}} \varepsilon l, 3 \mathrm{p} \pi_{\mathrm{u}} \varepsilon l$, and $3 \mathrm{~s} \sigma_{\mathrm{g}} \varepsilon l$ ionization continua. This amounts to $\sim 100000$ vibronic states. Since the ionization thresholds associated with the first three continua are the dominant ones, we have neglected interchannel couplings between those three continua and the continua associated with higher thresholds [19]. All other couplings, both dipolar (i.e., through the radiation-molecule potential $V(t)$ ) and electrostatic (i.e., through the molecular Hamiltonian) are taken into account in the calculations within the framework of the Born-Oppenheimer approximation.

To extract the dissociation probability into specific $\mathrm{H}(n l)$ states we have used the wellknown Barat-Lichten rules [29] to diabatically correlate the $\mathrm{H}_{2}\left(\mathrm{D}_{2}\right)$ resonant states and the $\mathrm{H}_{2}^{+}$ionization thresholds to the corresponding atomic states in the separate-atom limit. These correlations are given in table 1 . Note that some of these correlations are at variance with those used in the analysis of [16], which were obtained by assuming a diabatic behaviour between the Q1 and Q2 states and the singly excited configurations, and an adiabatic one within the Q1 and Q2 manifolds [15].

\section{Data and discussion}

Our data for both experiment and theory are shown in figures 2-5 in conjunction with the data of $[12,14,16]$. Because our experimental data are not absolute, we have normalized all our data sets to the theoretical value at $34 \mathrm{eV}$. This energy was picked for normalization because the Ly $\alpha$ data exhibit a maximum cross section in this region, and cascading effects are expected to be minimal (see below). In the case of the $\mathrm{H} \alpha$ excitation functions, the theoretically predicted $3 p$ contributions correspond to that fraction of the $3 p$ state that decays into the $2 \mathrm{~s}$ level with a branching ratio of $12 \%$. The indicated uncertainty in our data is due primarily to variations in the normalized intensities from run to run. Statistical noise and systematic uncertainties in the calibration of the photodiode used to detect the incident photon flux are small compared to these fluctuations. The agreement between our experiment and theory and the other experimental data is at best qualitative. The variation of the calculated Ly- $\alpha \mathrm{H}_{2}$ cross section with photon energy is in somewhat better agreement with the present measurements 
Table 1. Barat-Lichten rules [29] that correlate the separate-atom limit to the $\mathrm{H}_{2}$ doubly excited states and the $\mathrm{H}_{2}^{+}$ionization thresholds included in the theoretical calculations; (a) doubly excited states; (b) ionization thresholds. For each Q state, only the dominant doubly excited configuration is indicated (see [25] for details).

\begin{tabular}{|c|c|c|c|c|c|c|c|c|c|}
\hline \multicolumn{2}{|l|}{$\overline{\mathrm{Q}_{1}{ }^{1} \Sigma_{\mathrm{u}}^{+}}$} & \multicolumn{2}{|l|}{$\mathrm{Q}_{1}{ }^{1} \Pi_{\mathrm{u}}$} & \multicolumn{2}{|l|}{$\mathrm{Q}_{2}{ }^{1} \Sigma_{\mathrm{u}}^{+}$} & \multicolumn{4}{|l|}{$\mathrm{Q}_{2}{ }^{1} \Pi_{\mathrm{u}}$} \\
\hline \multicolumn{10}{|c|}{ (a) Doubly excited states } \\
\hline \multirow{2}{*}{\multicolumn{2}{|c|}{$\begin{array}{l}\mathrm{H}_{2}{ }^{* *}\left(2 \mathrm{p} \sigma_{\mathrm{u}}, 2 \mathrm{~s} \sigma_{\mathrm{g}}\right) \rightarrow \mathrm{H}(1 \mathrm{~s})+\mathrm{H}(2 \mathrm{~s}) \\
\mathrm{H}_{2}{ }^{* *}\left(2 \mathrm{p} \sigma_{\mathrm{u}}, 3 \mathrm{~d} \sigma_{\mathrm{g}} \rightarrow \mathrm{H}(1 \mathrm{~s})+\mathrm{H}(2 \mathrm{p})\right. \\
\mathrm{H}_{2}{ }^{* * *}\left(2 \mathrm{p} \sigma_{\mathrm{u}}, 3 \mathrm{~s} \sigma_{\mathrm{g}}\right) \rightarrow \mathrm{H}(1 \mathrm{~s})+\mathrm{H}(3 \mathrm{~s}) \\
\mathrm{H}_{2}{ }^{* * *}\left(2 \mathrm{p} \sigma_{\mathrm{u}}, 4 \mathrm{~d} \sigma_{\mathrm{g}}\right) \rightarrow \mathrm{H}(1 \mathrm{~s})+\mathrm{H}(3 \mathrm{p}) \\
\mathrm{H}_{2}{ }^{* * *}\left(2 \mathrm{p} \sigma_{\mathrm{u}}, 5 \mathrm{~g} \sigma_{\mathrm{g}}\right) \rightarrow \mathrm{H}(1 \mathrm{~s})+\mathrm{H}(3 \mathrm{~d}) \\
\mathrm{H}_{2}{ }^{* *}\left(2 \mathrm{p} \sigma_{\mathrm{u}}, 4 \mathrm{~s} \sigma_{\mathrm{g}}\right) \rightarrow \mathrm{H}(1 \mathrm{~s})+\mathrm{H}(n=4)\end{array}$}} & \multicolumn{2}{|c|}{$\begin{array}{l}\mathrm{H}_{2}^{* *}\left(2 \mathrm{p} \sigma_{\mathrm{u}}, 3 \mathrm{~d} \pi \mathrm{g}\right) \rightarrow \mathrm{H}(1 \mathrm{~s})+\mathrm{H}(2 \mathrm{p}) \\
\mathrm{H}_{2}{ }^{* *}\left(2 \mathrm{p} \sigma_{\mathrm{u}}, 4 \mathrm{~d} \pi \mathrm{g}\right) \rightarrow \mathrm{H}(1 \mathrm{~s})+\mathrm{H}(3 \mathrm{p}) \\
\mathrm{H}_{2}{ }^{* *}\left(2 \mathrm{p} \sigma_{\mathrm{u}}, 5 \mathrm{~g} \pi \mathrm{g}\right) \rightarrow \mathrm{H}(1 \mathrm{~s})+\mathrm{H}(3 \mathrm{~d}) \\
\mathrm{H}_{2}^{* *}\left(2 \mathrm{p} \sigma_{\mathrm{u}}, 5 \mathrm{~d} \pi_{\mathrm{g}}\right) \rightarrow \mathrm{H}(1 \mathrm{~s})+\mathrm{H}(n=4) \\
\mathrm{H}_{2}^{* *}\left(2 \mathrm{p} \sigma_{\mathrm{u}}, 6 \mathrm{~g} \pi_{\mathrm{g}}\right) \rightarrow \mathrm{H}(1 \mathrm{~s})+\mathrm{H}(n=4) \\
\mathrm{H}_{2}{ }^{* *}\left(2 \mathrm{p} \sigma_{\mathrm{u}}, 7 \mathrm{i} \pi_{\mathrm{g}}\right) \rightarrow \mathrm{H}(1 \mathrm{~s})+\mathrm{H}(n=4)\end{array}$} & \multicolumn{2}{|c|}{$\begin{array}{l}\mathrm{H}_{2}^{* *}\left(2 \mathrm{p} \pi_{\mathrm{u}}, 3 \mathrm{~d} \pi_{\mathrm{g}}\right) \rightarrow \mathrm{H}(2 \mathrm{p})+\mathrm{H}(2 \mathrm{p}) \\
\mathrm{H}_{2}{ }^{* *}\left(2 \mathrm{p} \pi_{\mathrm{u}}, 4 \mathrm{~d} \pi_{\mathrm{g}}\right) \rightarrow \mathrm{H}(2 \mathrm{p})+\mathrm{H}(3 \mathrm{p}) \\
\mathrm{H}_{2}{ }^{* *}\left(2 \mathrm{p} \pi_{\mathrm{u}}, 5 \mathrm{~d} \pi_{\mathrm{g}}\right) \rightarrow \mathrm{H}(2 \mathrm{p})+\mathrm{H}(n=4) \\
\mathrm{H}_{2}{ }^{* *}\left(2 \mathrm{p} \pi_{\mathrm{u}}, 5 \mathrm{~g} \pi_{\mathrm{g}}\right) \rightarrow \mathrm{H}(2 \mathrm{p})+\mathrm{H}(3 \mathrm{~d}) \\
\mathrm{H}_{2}{ }^{* *}\left(2 \mathrm{p} \pi_{\mathrm{u}}, 6 \mathrm{~d} \pi_{\mathrm{g}}\right) \rightarrow \mathrm{H}(2 \mathrm{p})+\mathrm{H}(n=5) \\
\mathrm{H}_{2}{ }^{* *}\left(2 \mathrm{p} \pi_{\mathrm{u}}, 6 \mathrm{~g} \pi_{\mathrm{g}}\right) \rightarrow \mathrm{H}(2 \mathrm{p})+\mathrm{H}(n=4)\end{array}$} & \multicolumn{4}{|c|}{$\begin{array}{l}\mathrm{H}_{2}^{* *}\left(2 \mathrm{p} \pi_{\mathrm{u}}, 2 \mathrm{~s} \sigma_{\mathrm{g}}\right) \rightarrow \mathrm{H}(2 \mathrm{p})+\mathrm{H}(2 \mathrm{~s}) \\
\mathrm{H}^{* * *}\left(2 \mathrm{p} \pi_{\mathrm{u}}, 3 \mathrm{~d} \sigma_{\mathrm{g}}\right) \rightarrow \mathrm{H}(2 \mathrm{p})+\mathrm{H}(2 \mathrm{p}) \\
\mathrm{H}^{* * *}\left(2 \mathrm{p} \pi_{\mathrm{u}}, 3 \mathrm{~s} \sigma_{\mathrm{g}}\right) \rightarrow \mathrm{H}(2 \mathrm{p})+\mathrm{H}(3 \mathrm{~s}) \\
\mathrm{H}^{* *}\left(2 \mathrm{p} \pi_{\mathrm{u}}, 3 \mathrm{~s} \sigma_{\mathrm{g}}\right) \rightarrow \mathrm{H}(2 \mathrm{p})+\mathrm{H}(3 \mathrm{~s}) \\
\mathrm{H}^{* *}\left(2 \mathrm{p} \pi_{\mathrm{u}}, 4 \mathrm{~s} \sigma_{\mathrm{g}}\right) \rightarrow \mathrm{H}(2 \mathrm{p})+\mathrm{H}(n=4) \\
\mathrm{H}^{* *}\left(2 \mathrm{p} \pi_{\mathrm{u}}, 5 \mathrm{~d} \sigma_{\mathrm{g}}\right) \rightarrow \mathrm{H}(2 \mathrm{p})+\mathrm{H}(n=4) \\
\mathrm{H}_{2}{ }^{* *}\left(2 \mathrm{p} \pi_{\mathrm{u}}, 5 \mathrm{~g} \sigma_{\mathrm{g}}\right) \rightarrow \mathrm{H}(2 \mathrm{p})+\mathrm{H}(3 \mathrm{~d})\end{array}$} \\
\hline & & \multicolumn{4}{|c|}{ (b) Ionization thresholds } & & & & \\
\hline $\mathrm{H}_{2}^{+}\left(1 \mathrm{~s} \sigma_{\mathrm{g}}\right)$ & $\mathbf{H}_{2}^{+}\left(2 \mathrm{p} \sigma_{\mathrm{u}}\right)$ & $\mathrm{H}_{2}^{+}\left(2 \mathrm{p} \pi_{\mathrm{u}}\right)$ & $\mathrm{H}_{2}^{+}\left(2 \mathrm{~s} \sigma_{\mathrm{g}}\right)$ & $\mathrm{H}_{2}^{+}\left(3 \mathrm{p} \sigma_{\mathrm{u}}\right)$ & $\mathrm{H}_{2}^{+}(3 \mathrm{~d} \sigma \mathrm{g})$ & $\mathrm{H}_{2}^{+}\left(3 \mathrm{~d} \pi_{\mathrm{g}}\right)$ & $\mathrm{H}_{2}^{+}\left(3 \mathrm{~d} \delta_{\mathrm{g}}\right)$ & $\mathrm{H}_{2}^{+}\left(3 \mathrm{p} \pi_{\mathrm{u}}\right)$ & $\mathrm{H}_{2}^{+}\left(3 \mathrm{~s} \sigma \sigma_{\mathrm{g}}\right)$ \\
\hline
\end{tabular}




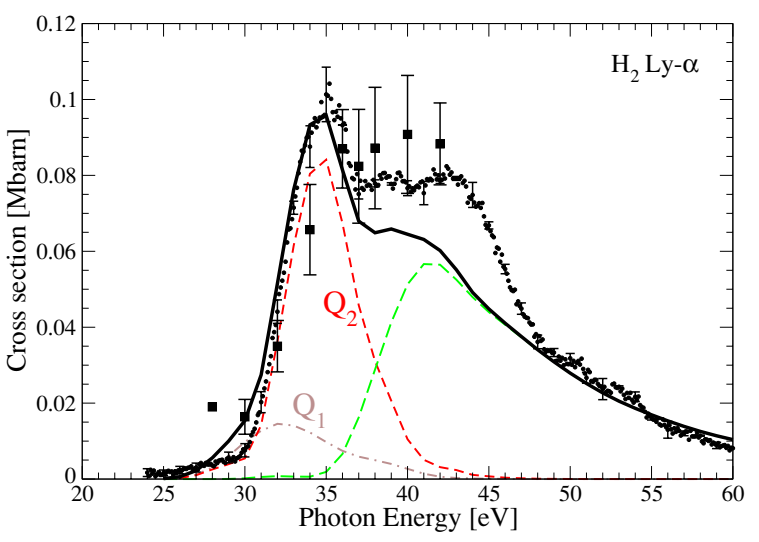

Figure 2. Excitation functions for Lyman- $\alpha$ photoemission resulting from $\mathrm{H}_{2}$ photodissociation by linearly polarized light. Circles: present experimental results normalized to theory at $34 \mathrm{eV}$; squares: absolute results of Glass-Maujean et al [12]. Theory: thick black curve, total H(2p) cross section; dashed-dotted line, contribution of Q1 doubly excited states (channel (3)); short dashed line, contribution of Q2 doubly excited states (channel (3)); long dashed line, non-resonant contribution corresponding to the dissociative ionization process (channel (2)).

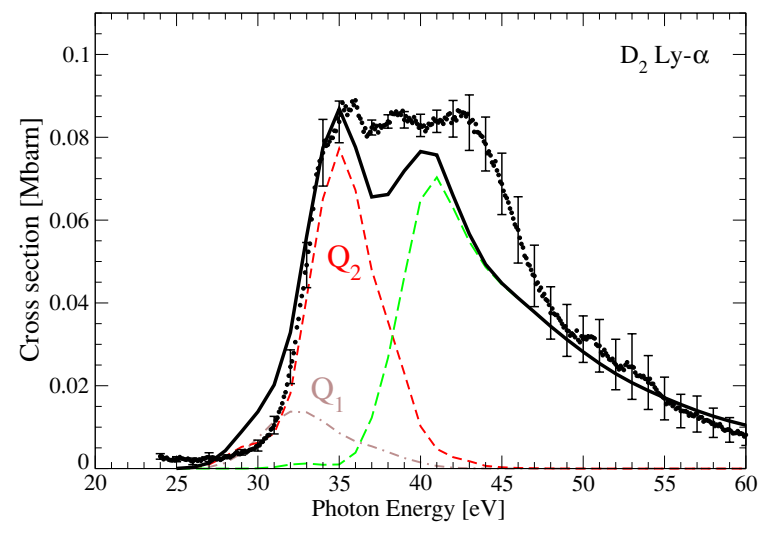

Figure 3. The same as in figure 2 but for $\mathrm{D}_{2}$ molecules.

than with those of [12]. Our calculated values are comparable to the absolute ones reported in the latter reference. The most significant disagreements occur with the H $\alpha$ results, where the high-energy secondary maxima observed experimentally are, in comparison with the lowenergy peak, significantly larger than in the calculated cross sections. It is important to note that channel (3) resonant $\mathrm{Q}_{3}\left(2 \mathrm{~s} \sigma_{\mathrm{g}}\right)$ doubly excited state excitation-which is required for reasonable heuristic fits to the $\mathrm{H} \alpha$ data $[6,16]$ - is predicted to be small-to-negligible on the basis of our ab initio theory (see also [5]).

It can be seen that the $\mathrm{H}_{2}$ and $\mathrm{D}_{2} \mathrm{Ly} \alpha$ spectra are significantly different. Two effects can explain this observation. First, $\mathrm{D}_{2}$ dissociates more slowly than $\mathrm{H}_{2}$ (due to the different mass), so that the time available for autoionization is slightly larger and, consequently, the dissociation yield associated with the doubly-excited states is smaller. Second, the 'effective' Franck-Condon region in $\mathrm{D}_{2}$ is narrower than in $\mathrm{H}_{2}$ and, therefore, fewer doubly excited states are efficiently populated, leading again to a reduction in the dissociation yield associated with the doubly excited states. Both effects explain why the Ly $\alpha$ resonant contribution is relatively 


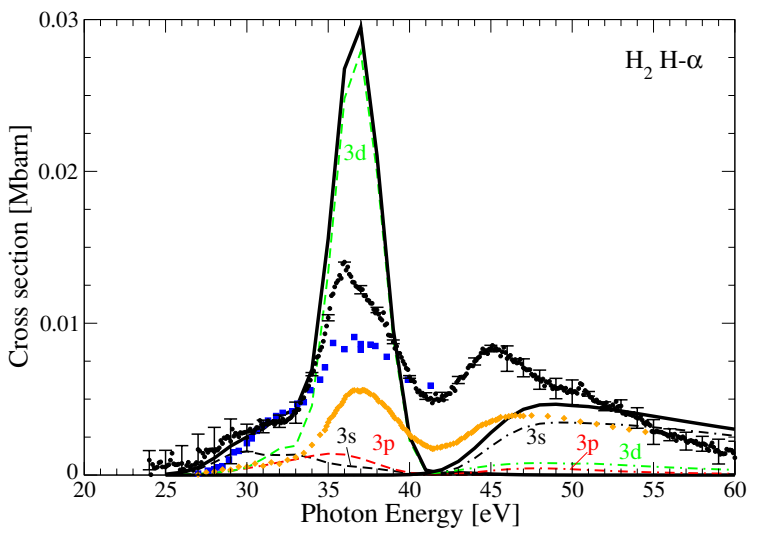

Figure 4. Excitation functions for $\mathrm{H}-\alpha$ photoemission resulting from $\mathrm{H}_{2}$ photodissociation by linearly polarized light. Experiment: circles, present results normalized to theory at $34 \mathrm{eV}$; squares, absolute results of Glass-Maujean et al [15]; diamonds, absolute results of Garcia et al [16]. Theoretical fluorescence cross section equals the combination of production cross sections $\mathrm{H}(3 \mathrm{~s})+0.12 \mathrm{H}(3 \mathrm{p})+\mathrm{H}(3 \mathrm{~d})$ (thick black line); dashed lines, contribution from doubly excited states; dashed-dotted lines, non-resonant contribution corresponding to the dissociative ionization processes. Black: $\mathrm{H}(3 \mathrm{~s})$; red: $0.12 \mathrm{H}(3 \mathrm{p})$; green: $\mathrm{H}(3 \mathrm{~d})$.

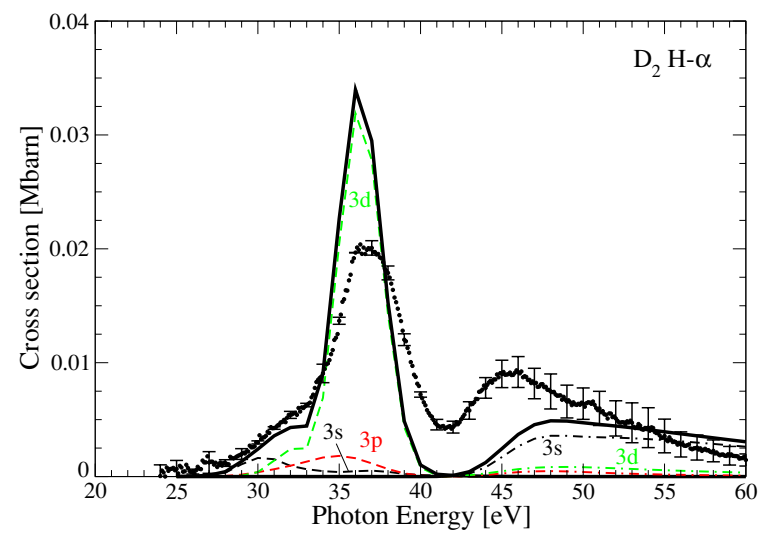

Figure 5. The same as in figure 4 but for $D_{2}$ molecules.

larger in $\mathrm{H}_{2}$ than in $\mathrm{D}_{2}$ (see figures 2 and 3). However, one must also take into account that, due to interference effects between resonant and non-resonant processes, the ionization profile does not necessarily follow the Franck-Condon behaviour [2]. Although the latter effect does not seem to modify the 'expected' behaviour in the Ly $\alpha$ case, the similarity of the H $\alpha$ spectra in $\mathrm{H}_{2}$ and $\mathrm{D}_{2}$ (see figures 4 and 5) suggests that this is not the general case.

None of the experimental data sets shown in figures $2-5$ have been corrected for cascading contributions or the effects of l-state-dependent detection efficiency [16]. The latter problem can arise if the volume over which photons must be emitted in order to be detected is comparable in length scale to the distance an emitting photofragment travels in a fluorescence lifetime. Using a typical dissociation energy of $10-15 \mathrm{eV}$ (which is shared equally between the two heavy photofragments) for the processes considered here, the emitting hydrogen atoms will be travelling at $\sim 35 \mathrm{~km} \mathrm{~s}^{-1}$. Thus a Ly $\alpha$ lifetime $(1.6 \mathrm{~ns})$ corresponds to a distance of $\sim 0.06 \mathrm{~mm}$. The $\mathrm{H}(n=3)$ states have lifetimes of $158,5.3$ and $15.5 \mathrm{~ns}$ for $l=0,1$ and 2 , respectively. 


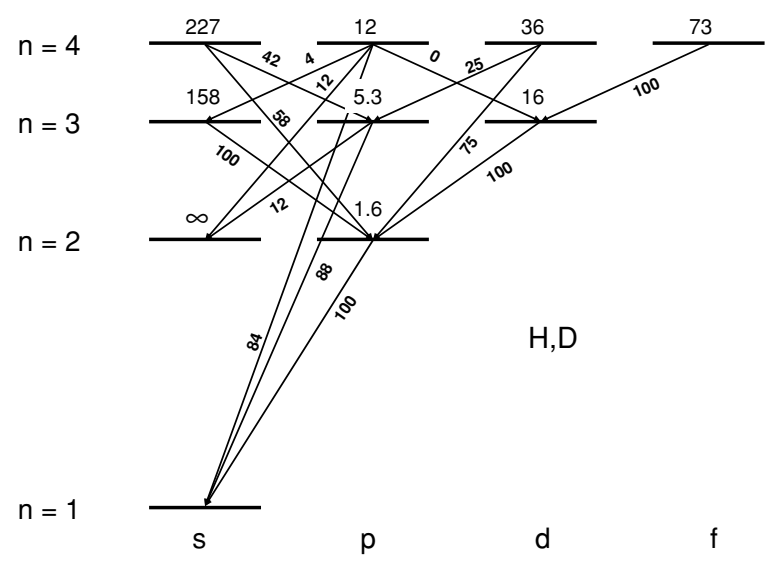

Figure 6. Partial Grotrian diagram of the $\mathrm{H}$ or $\mathrm{D}$ atom indicating state lifetimes and fluorescence branching ratios.

Thus only the $3 \mathrm{~s}$ state can be expected to exhibit any cut-off of volume detection efficiency. Garcia et al [16] quote a sensitive solid angle corresponding to a transverse area of the order of $(2 \mathrm{~mm})^{3}$, and thus expect significantly reduced sensitivity for $3 \mathrm{~s}-2 \mathrm{p}$ emission only. Our detection solid angles (for a purely geometric projection in the case of the Ly $\alpha$ channeltron and an effective photocathode image size at the interaction region in the case of $\mathrm{H} \alpha$ ) correspond to transverse areas at the interaction region of the order of $(\sim 1.3 \mathrm{~cm})^{2}$. Thus we expect perhaps a $1 / 3$ reduction in our sensitivity to $3 s-2 p$ emission. Having said this, the theoretical calculations we have performed indicate that $3 \mathrm{~s}$ fluorescence is important in the regions below $34 \mathrm{eV}$ for $\mathrm{H}_{2}$ and $33 \mathrm{eV}$ for $\mathrm{D}_{2}$, and above $42 \mathrm{eV}$ in both cases, in marked disagreement with the conclusions of Garcia et al ([16], their figure 5) and Glass-Maujean et al ([15], their figure 4(a)). Since our experimental cross sections have enhanced values in the middle range between 33 and $43 \mathrm{eV}$ compared with theory, a reduced sensitivity to $3 \mathrm{~s}$ fluorescence cannot explain the different shape of the two data sets.

Cascading contributions represent a more serious obstacle when it comes to interpreting the data and making meaningful comparisons between experiment and theory. A Grotrian diagram for $\mathrm{H}(n=1-4)$, shown in figure 6 , indicates the lifetimes and branching ratios for the various states of relevance for this discussion. For the solid angles that our detectors viewed in this experiment, it is safe to assume that all the population of the $4 \mathrm{f}$ state will ultimately be detected as both $\mathrm{H} \alpha$ and $\mathrm{Ly} \alpha$ fluorescence, while the $4 \mathrm{~d}$ state population will result through cascade contributions essentially only in Ly $\alpha$ light because of the large 1s-3p branching ratio. Cascading from the $4 \mathrm{p}$ state can reasonably be ignored, while contributions from the $4 \mathrm{~s}$ state will result in both $\mathrm{H} \alpha$ and $\mathrm{Ly} \alpha$ fluorescence, albeit with a significantly reduced contribution due to the long lifetime (227 ns) of this state. Making reasonable assumptions about the geometric diminishment of sensitivity for relatively long-lived states, we estimate that the fluorescence intensity we detect, $I=S+C$, where $S$ is the direct signal and $C$ is the cascading contribution, can be characterized for $\mathrm{H}_{2}$ targets by

$$
\begin{aligned}
& S(\operatorname{Ly} \alpha) \propto \sigma_{2 \mathrm{p}}, \\
& C(\operatorname{Ly} \alpha) \propto\left(\sigma_{3 \mathrm{~d}}+\sigma_{4 \mathrm{f}}+0.75 \sigma_{4 \mathrm{~d}}+0.26 \sigma_{4 \mathrm{~s}}+0.63 \sigma_{3 \mathrm{~s}}\right), \\
& S(\mathrm{H} \alpha) \propto\left(\sigma_{3 \mathrm{~d}}+0.12 \sigma_{3 \mathrm{p}}+\sigma_{3 \mathrm{~s}}\right),
\end{aligned}
$$

and

$$
C(\mathrm{H} \alpha) \propto \sigma_{4 \mathrm{f}},
$$




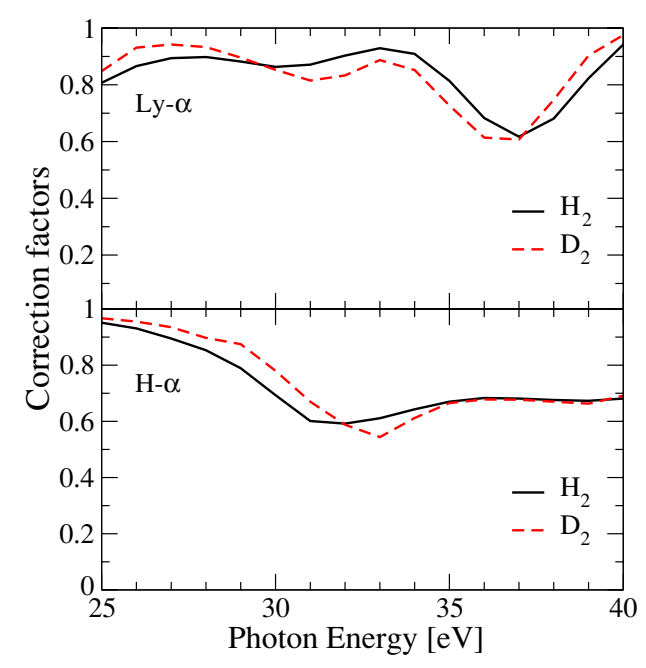

Figure 7. The ratio of direct fluorescence signal ( $S$; see text equation (5)) to total signal including cascading contribution $(I+S)$, or cascading 'correction factor' versus incident photon energy. Data from theoretical calculations for resonant excitation of higher lying states only.

where $\sigma$ is a production cross section. In the case of deuterium, for which the heavy photofragments have a speed of 0.71 that of the equivalent $\mathrm{H}_{2}$ fragments, equation ( $5 b$ ) becomes

$$
C(\operatorname{Ly} \alpha) \propto\left(\sigma_{3 \mathrm{~d}}+\sigma_{4 \mathrm{f}}+0.75 \sigma_{4 \mathrm{~d}}+0.37 \sigma_{4 \mathrm{~s}}+0.76 \sigma_{3 s}\right) .
$$

Using theoretical estimates of the $n=3$ and $n=4$ production cross sections, we can thus estimate the cascading contribution to our $\mathrm{Ly} \alpha$ and $\mathrm{H} \alpha$ fluorescence signals. These are shown in figure 7. Since non-resonant dissociative channels leading to $\mathrm{H}(n=4)$ are not included in the calculations (see table 1), our theoretical estimates of the $\mathrm{H}(n=4)$ populations are only valid in the resonant region. Furthermore, since only the six lowest doubly excited states of each symmetry are included (see table 1$)$, the calculated $\mathrm{H}(n=4)$ populations are not expected to be accurate to better than a factor of 2 in the resonant region. It is expected that $n=5$ and higher lying populations will produce some cascading signal, with the $5 \mathrm{~d}$ state contributing the most significantly, through a direct transition to the $2 p$ state. The lifetimes of the $5 \mathrm{~s}$ and $5 \mathrm{~g}$ states are 352 and $235 \mathrm{~ns}$, respectively, and are not expected to contribute significantly. Nonetheless, the cascading estimates shown in figure 7 should be taken as upper bounds and should be qualitatively correct in the resonant region.

In the case of both the $\mathrm{H}_{2}$ and $\mathrm{D}_{2} \mathrm{Ly}-\alpha$ data, it is apparent that at least some of the discrepancy between experiment and theory above $34 \mathrm{eV}$ can be explained in terms of cascading contributions. The normalized experimental data points lie almost exclusively above the theory in this energy range, with the deviation becoming increasingly significant above $36 \mathrm{eV}$, where the cascading correction factor due to resonant processes is the largest. A non-resonant cascade contribution, not calculated here, and not included in the data of figure 7, could account for the even larger discrepancies above $38-39 \mathrm{eV}$.

Since the theoretical calculations indicate that the contribution of the Q3 autoionizing states is much smaller than that of the Q1 and Q2 ones, discrepancies between experiment and theory in the 35-48 eV energy range cannot be explained in terms of the populations of these higher lying $\mathrm{Q}$ states. 
One would expect that application of an electric field to the interaction region might affect cascade contributions to the fluorescence signal by mixing higher-lying excited states. Indeed, this technique may prove effective for quantifying these effects. We note that in a preliminary crude experiment, we applied weak $\left(<50 \mathrm{~V} \mathrm{~cm}^{-1}\right)$ electric fields to the target volume by means of a single electrode, and saw no significant change in the excitation functions we measured.

\section{Conclusions}

We have compared in this paper a precise, comprehensive excitation-function data set for photodissociation of the fundamental $\mathrm{H}_{2}$ and $\mathrm{D}_{2}$ systems with a state-of-the-art ab initio theory. Given the prototypical nature of this chemical system, it is particularly troubling that none of the experimental data agree well with each other, and that none are in full agreement with the theory. The most significant disagreement between experiment and theory centres on whether excitation of the higher lying Q3 and Q4 states is important, and the level at which the $3 \mathrm{~s}$ states contribute to the $\mathrm{H}-\alpha$ signal. It is apparent that significantly more work needs to be done by both experimentalists and theorists to understand at a basic level how a doubly excited hydrogen or deuterium molecule falls apart. Effects due to cascading must be quantified, and the troubling discrepancy between the available data sets needs to be resolved.

\section{Acknowledgments}

We wish to thank O Yenen, D H Jaecks, T A Rescigno, A E Orel and M W McCurdy for useful conversations. This work has been funded by the DOE through use of the ALS, the NSF through grants PHY-0354946 and PHY-0321055, DGI Project BFM2003-00194 and the European COST Action D26/0002/02. We thank the CCC-UAM (Madrid, Spain) for computer time. JEF wishes to acknowledge support from the University of Newcastle Outside Studies Program and JLS-V the Comisión de Servicios granted by the Universidad de Antioquia and funding by CIEN (UdeA) and the Colciencias Agency (Colombia). One of us (HG) was supported by the Office of Science, Office of Basic Energy Sciences of the US DOE. The Lawrence Berkeley National Laboratory is operated for the US DOE under contract DE-AC02-05CH11231.

\section{References}

[1] Kouchi N, Ukai M and Hatano Y 1997 J. Phys. B: At. Mol. Opt. Phys. 302319

[2] Sánchez I and Martín F 1999 Phys. Rev. Lett. 823775

[3] Borges I Jr and Bielschowsky C E 2000 J. Phys. B: At. Mol. Opt. Phys. 331713

[4] Borges I Jr and Bielschowsky C E 2001 Chem. Phys. Lett. 342411

[5] Aoto T, Hikosaka Y, Hall R I, Ito K, Fernández J and Martín F 2004 Chem. Phys. Lett. 389145

[6] Glass-Maujean M and Schmoranzer H 2005 J. Phys. B: At. Mol. Opt. Phys. 381093

[7] Vanroose W, Martín F, Rescigno T N and McCurdy C W 2005 Science 3101787

[8] Fantz U 2005 Atomic and molecular data and their applications Proc. Joint Meeting of the 14th Int. Toki Conf. on Plasma Physics and Controlled Nuclear Fusion and the 4th Int. Conf. on Atomic and Molecular Data and their Applications ed T Kato, D Kato and H Funaba (AIP Conf. Proc. vol 771)

[9] Ferland G J 2003 Annu. Rev. Astron. Astrophys. 41517

[10] Liang M-C, Parkinson C D, Lee A Y-T, Yung Y L and Seager S 2003 Astrophys. J. 596 L247

[11] Weber T et al 2004 Nature 431437

[12] Glass-Maujean M, Klumpp S, Werner L, Ehresmann A and Schmoranzer H 2004 J. Phys. B: At. Mol. Opt. Phys. 372677

[13] Glass-Maujean M 1986 J. Chem. Phys. 854830

[14] Glass-Maujean M 1988 J. Chem. Phys. 892839 
[15] Glass-Maujean M, Frohlich H and Martin P 1995 Phys. Rev. A 524622

[16] Melero García E, Álvarez Ruiz J, Menmuir S, Rachlew E, Erman P, Kivimäki A, Glass-Maujean M, Richter R and Coreno M 2006 J. Phys. B: At. Mol. Opt. Phys. 39205

[17] Arai S, Kamosaka T, Ukai M, Shinsaka K, Hatano Y, Ito Y, Koizumi H, Yagishita A, Ito K and Tanaka K 1988 J. Chem. Phys. $\mathbf{8 8} 3016$

[18] Odagiri T, Murata M, Kato M and Kouchi N 2004 J. Phys. B: At. Mol. Opt. Phys. 373909

[19] Sanz-Vicario J L, Bachau H and Martín F 2006 Phys. Rev. A 73033410

[20] McLaughlin K W, Yenen O, Jaecks D H, Gay T J, Sant'Anna M M, Calabrese D and Jordan-Thaden B 2002 Phys. Rev. Lett. 88123003

[21] Clout P N and Heddle D W O 1969 J. Opt. Soc. Am. 59715

[22] Glass-Maujean M, Lauer S, Liebel H and Schmoranzer H 2000 J. Phys. B: At. Mol. Opt. Phys. 334593

[23] Humphrey I 2000 Meas. Sci. Technol. 111193 and references therein

[24] Martín F 1999 J. Phys. B: At. Mol. Opt. Phys. 32 R197

[25] Latimer C J, Dunn K F, O’Neill F P, McDonald M A and Kouchi N 1995 J. Chem. Phys. 102722

[26] Sánchez I and Martín F 1997 J. Chem. Phys. 1067720

[27] Sánchez I and Martín F 1999 J. Chem. Phys. 1106702

[28] Fernández J and Martín F 2001 J. Phys. B: At. Mol. Opt. Phys. 344141

[29] Barat M and Lichten W 1972 Phys. Rev. A 6211 